PROCEEDINGS OF THE

AMERICAN MATHEMATICAL SOCIETY

Volume 131, Number 3, Pages 953-961

S 0002-9939(02)06736-9

Article electronically published on July 17, 2002

\title{
A CHARACTERIZATION OF COMPACTLY GENERATED METRIC GROUPS
}

\author{
HIROSHI FUJITA AND DMITRI SHAKHMATOV \\ (Communicated by Alan Dow)
}

\begin{abstract}
Recall that a topological group $G$ is: (a) $\sigma$-compact if $G=\bigcup\left\{K_{n}\right.$ : $n \in \mathbf{N}\}$ where each $K_{n}$ is compact, and (b) compactly generated if $G$ is algebraically generated by some compact subset of $G$. Compactly generated groups are $\sigma$-compact, but the converse is not true: every countable nonfinitely generated discrete group (for example, the group of rational numbers or the free (Abelian) group with a countable infinite set of generators) is a counterexample. We prove that a metric group $G$ is compactly generated if and only if $G$ is $\sigma$-compact and for every open subgroup $H$ of $G$ there exists a finite set $F$ such that $F \cup H$ algebraically generates $G$. As a corollary, we obtain that a $\sigma$-compact metric group $G$ is compactly generated provided that one of the following conditions holds: (i) $G$ has no proper open subgroups, (ii) $G$ is dense in some connected group (in particular, if $G$ is connected itself), (iii) $G$ is totally bounded (= subgroup of a compact group). Our second major result states that a countable metric group is compactly generated if and only if it can be generated by a sequence converging to its identity element (eventually constant sequences are not excluded here). Therefore, a countable metric group $G$ can be generated by a (possibly eventually constant) sequence converging to its identity element in each of the cases (i), (ii) and (iii) above. Examples demonstrating that various conditions cannot be omitted or relaxed are constructed. In particular, we exhibit a countable totally bounded group which is not compactly generated.
\end{abstract}

If $G$ is a group and $X \subseteq G$, then we use $\langle X\rangle$ to denote the smallest subgroup of $G$ which contains $X$. A set $X$ algebraically generates $G$ if $G=\langle X\rangle$. Recall that a topological group $G$ is:

(i) $\sigma$-compact if $G=\bigcup\left\{K_{n}: n \in \mathbf{N}\right\}$ where each $K_{n}$ is compact, and

(ii) compactly generated if $G=\langle K\rangle$ for some compact subset $K$ of $G$.

Obviously, each compactly generated group is $\sigma$-compact. The converse is not true in general: any countable nonfinitely generated discrete group (for example, the group of rational numbers or the free (Abelian) group with a countable infinite set of generators) is a counterexample. However, Pestov [15] established that every $\sigma$-compact topological group is topologically isomorphic to a closed subgroup of some compactly generated group.

Received by the editors May 5, 1997 and, in revised form, August 17, 2001 and October 17, 2001.

2000 Mathematics Subject Classification. Primary 54H11, 22A05.

Key words and phrases. Compact, compactly generated, topological group, metric space, metric, convergent sequence.

(C)2002 American Mathematical Society 
The primary goal of this paper is to give a characterization of metric groups which are compactly generated (Corollary 11). It follows from our characterization that both connected and totally bounded $\sigma$-compact metric groups are compactly generated (Corollaries [15] and (16). We also show in Theorem[17 that a countable metric group is compactly generated if and only if it can be generated by a sequence converging to its identity element (eventually constant sequences are not excluded here).

\section{Preliminaries}

We start by stating explicitly a folklore fact which will be used in our proofs:

Lemma 1. Let $\left\{U_{n}: n \in \mathbf{N}\right\}$ be a local base at point e of a topological space $G$ such that $\overline{U_{n+1}} \subseteq U_{n}$ for all $n$. Suppose that $\left\{L_{n}: n \in \mathbf{N}\right\}$ is a sequence of subsets of $G$ such that:

(i) $L_{n}$ is compact, and

(ii) $L_{n} \subseteq \overline{U_{n}}$.

Then $L=\bigcup\left\{L_{n}: n \in \mathbf{N}\right\} \cup\{e\}$ is compact. Moreover, if each $L_{n}$ is finite, then $L$ is a (possibly eventually constant) sequence converging to $e$.

Lemma 2. Let $G$ be a dense subgroup of a topological group $G^{\prime}$. Then for every open subgroup $H$ of $G$ there exists an open subgroup $H^{\prime}$ of $G^{\prime}$ with $H^{\prime} \cap G=H$.

Proof. For a set $A \subseteq G^{\prime}$ we use $\bar{A}$ to denote the closure of $A$ in $G^{\prime}$. Define $H^{\prime}$ to be the union of all open subsets $V$ of $G^{\prime}$ such that $V \cap G \subseteq H$. Clearly, $H^{\prime}$ is an open subset of $G$ and $H^{\prime} \cap G=H$. We claim that $H^{\prime}$ is a subgroup of $G$. Indeed, assume that $g, h \in H^{\prime}$. By our definition of $H^{\prime}$, there exist sets $U$ and $V$, open in $G^{\prime}$, such that $g \in U, h \in V, U \cap G \subseteq H$ and $V \cap G \subseteq H$. Since $G$ is dense in $G^{\prime}$, $\overline{U \cap G}=\bar{U}$ and $\overline{V \cap G}=\bar{V}$. Thus

$$
U V \subseteq \bar{U} \cdot \bar{V}=\overline{U \cap G} \cdot \overline{V \cap G} \subseteq \overline{(U \cap G) \cdot(V \cap G)},
$$

where the last inclusion follows from the continuity of multiplication in $G^{\prime}$. Therefore,

$$
(U V) \cap G \subseteq \overline{(U \cap G) \cdot(V \cap G)} \cap G \subseteq \overline{H \cdot H} \cap G=\bar{H} \cap G=H,
$$

where the last equality is based on the fact that $H$ is closed in $G$, being an open subgroup of $G$ [10, Ch. II, Theorem 5.5]. Since $U V$ is open in $G^{\prime}$, we conclude that $g h \in U V \subseteq H^{\prime}$.

Assume now that $g \in H^{\prime}$. Then there exists a set $U$ open in $G^{\prime}$ such that $g \in U$ and $U \cap G \subseteq H$. Since $U^{-1}$ is open in $G^{\prime}$ and $U^{-1} \cap G=(U \cap G)^{-1} \subseteq H^{-1}=H$, it follows that $g^{-1} \in U^{-1} \subseteq H^{\prime}$.

Corollary 3. A dense subgroup of a connected group has no proper open subgroups.

Proof. An open subgroup of a topological group is also closed [10, Ch. II, Theorem $5.5]$, and so a connected group cannot have proper open subgroups. Now the result follows from Lemma 2

By [10, (7.9)] a locally bounded group (that is, a subgroup of a locally compact group) without proper open subgroups is a dense subgroup of a connected group, namely its completion. The converse of Corollary 3, however, does not hold in general. Indeed, Stevens [18] gave an example of a totally disconnected complete metric group $G$ without proper open subgroups. Since complete metric groups 
are closed in every group in which they are embedded, it follows that $G$ is not isomorphic to a dense subgroup of any connected topological group.

\section{RESUltS FOR METRIC GROUPS}

Theorem 4. If a $\sigma$-compact metric group $G$ contains a dense compactly generated subgroup, then $G$ itself is compactly generated.

Proof. Let $H$ be a dense subgroup of $G$ which is generated by some compact set $E$. Since $G$ is metric, we can choose a local base $\left\{U_{n}: n \in \mathbf{N}\right\}$ at $e$ consisting of symmetric neigbourhoods of $e$ such that $\overline{U_{n+1}} \subseteq U_{n}$ for each $n$. Since $G$ is $\sigma$-compact, there exists a sequence $\left\{K_{n}: n \in \mathbf{N}\right\}$ of compact subsets of $G$ with $G=\bigcup\left\{K_{n}: n \in \mathbf{N}\right\}$. Since $H$ is dense in $G$, we have $H U_{n}=G$ for all $n \in \mathbf{N}$. So for each $n \in \mathbf{N}$ there exists a finite subset $F_{n}$ of $H$ such that $F_{n} U_{n} \supseteq K_{n}$. If we set $L_{n}=\overline{U_{n}} \cap F_{n}^{-1} K_{n}$, then each $L_{n}$ is compact, and so, by Lemma 1 $L=\bigcup\left\{L_{n}: n \in \mathbf{N}\right\} \cup\{e\}$ is also compact. By our choice of $F_{n}, H L_{n} \supseteq F_{n} L_{n} \supseteq K_{n}$ for all $n$. Therefore, $H L \supseteq \bigcup\left\{H L_{n}: n \in \mathbf{N}\right\} \supseteq \bigcup\left\{K_{n}: n \in \mathbf{N}\right\}=G$. Since $H$ is generated by $E, G$ is generated by $E \cup L$. Since both $E$ and $L$ are compact, so is $E \cup L$. Therefore, $G$ is compactly generated.

Corollary 5. A $\sigma$-compact metric group which contains a dense finitely generated subgroup is compactly generated.

Proposition 6. For a topological group $G$ the following conditions are equivalent:

(i) for every nonempty open subset $V$ of $G$ there is a finite subset $F$ of $G$ such that $G=\langle F \cup V\rangle$,

(ii) for every open subgroup $H$ of $G$ there is a finite subset $F$ of $G$ such that $G=\langle F \cup H\rangle$.

Proof. The implication (i) $\rightarrow$ (ii) is obvious, so it remains only to prove that (ii) implies (i). Assume that (ii) holds and let $V$ be a nonempty open subset of $G$. Then $H=\langle V\rangle$ is an open subgroup of $G$, and so by (ii) there exists a finite set $F \subseteq G$ such that $G=\langle F \cup H\rangle=\langle F \cup\langle V\rangle\rangle=\langle F \cup V\rangle$.

Definition 7. We will say that a topological group $G$ is finitely generated modulo open sets provided that $G$ satisfies one of the equivalent conditions of Proposition 6]

Our next theorem establishes a simple necessary condition for a topological group to be compactly generated.

Theorem 8. If a topological group $G$ is compactly generated, then it is finitely generated modulo open sets.

Proof. Suppose that $G$ is generated by a compact set $K$. Let $H$ be an open subgroup of $G$. Then $\mathcal{H}=\{g H: g \in G\}$ is an open covering of $G$. Some finitely many classes, say $a_{1} H, \ldots, a_{n} H$, must cover $K$. Now note that $G=\langle F \cup H\rangle$, where $F=\left\{a_{1}, \ldots, a_{n}\right\}$.

Theorem 9. Let $G$ be a metric group and let $D$ be a countable subset of $G$. Assume that $G$ is finitely generated modulo open sets. Then $G$ contains a (possibly eventually constant) sequence $S$ converging to the identity e of $G$ such that $D \subseteq\langle S\rangle$. 
Proof. Let $D=\left\{d_{n}: n \in \mathbf{N}\right\}$ be an enumeration of $D$, and let $\left\{V_{n}: n \in \mathbf{N}\right\}$ be a local base at $e$ such that

$$
G=V_{0} \supseteq V_{1} \supseteq \cdots \supseteq V_{n} \supseteq \cdots
$$

If $V$ is a nonempty open subset of $G$, then $\langle V\rangle$ is an open subgroup of $G$. Therefore, by the hypothesis of our theorem we can fix, for each $n \in \mathbf{N}$, a finite set $F_{n}$ such that $G=\left\langle F_{n} \cup V_{n+1}\right\rangle$.

By induction on $n$ we will define a sequence $\left\{E_{n}: n \in \mathbf{N}\right\}$ of finite subsets of $G$ with the following properties:

(i $\left.\mathrm{i}_{n}\right) \subseteq V_{n}$,

(ii $\left.{ }_{n}\right) G=\left\langle E_{0} \cup E_{1} \cup \cdots \cup E_{n} \cup V_{n+1}\right\rangle$, and

$\left(\right.$ iii $\left._{n}\right) d_{n} \in\left\langle E_{0} \cup E_{1} \cup \cdots \cup E_{n}\right\rangle$.

To begin, note that the set $E_{0}=F_{0} \cup\left\{d_{0}\right\}$ satisfies all three conditions $\left(\mathrm{i}_{0}\right)-$ (iii $\left.{ }_{0}\right)$. Suppose that we have already defined finite sets $E_{0}, E_{1}, \ldots, E_{n-1}$ such that conditions $\left(\mathrm{i}_{0}\right), \ldots,\left(\mathrm{i}_{n-1}\right),\left(\mathrm{ii}_{0}\right), \ldots,\left(\mathrm{ii}_{n-1}\right)$ and $\left(\mathrm{iii}_{0}\right), \ldots,\left(\mathrm{iii}_{n-1}\right)$ are satisfied. Condition $\left(\mathrm{ii}_{n-1}\right)$ implies that

$$
F_{n} \cup\left\{d_{n}\right\} \subseteq\left\langle E_{0} \cup E_{1} \cup \cdots \cup E_{n-1} \cup V_{n}\right\rangle,
$$

and since the set $F_{n}$ is finite, we can find a finite set $E_{n} \subseteq V_{n}$ such that

$$
F_{n} \cup\left\{d_{n}\right\} \subseteq\left\langle E_{0} \cup E_{1} \cup \cdots \cup E_{n-1} \cup E_{n}\right\rangle .
$$

An easy check that $\left(\mathrm{i}_{n}\right)-\left(\mathrm{iii}_{n}\right)$ are satisfied is left to the reader.

From Lemma 1 and all $\left(\mathrm{i}_{n}\right)$ it follows that the set $S=\bigcup\left\{E_{n}: n \in \mathbf{N}\right\}$ forms a sequence converging to $e$. Finally, note that, by (iii $\left.{ }_{n}\right)$ for all $n, D \subseteq\langle S\rangle$.

Theorem 10. For a $\sigma$-compact metric group $G$ the following conditions are equivalent:

(i) $G$ is compactly generated,

(ii) $G$ is finitely generated modulo open sets.

Proof. The implication (i) $\rightarrow$ (ii) follows from Theorem 8 , Let us show that (ii) implies (i). Assume that for every open subgroup $H$ of $G$ there is a finite set $F$ such that $G=\langle F \cup H\rangle$. Since $G$ is $\sigma$-compact and metric, $G$ is separable. Let $D$ be any countable dense subset of $G$. Applying Theorem 9 we conclude that $G$ has a dense compactly generated subgroup. Theorem 4 now guarantees that $G$ itself is compactly generated.

Corollary 11. A metric group $G$ is compactly generated if and only if $G$ is $\sigma$ compact and finitely generated modulo open sets.

Corollary 12. An Abelian metric group $G$ is compactly generated if and only if $G$ is $\sigma$-compact and all discrete quotient groups of $G$ are finitely generated.

Now we proceed with describing particular classes of topological groups which are compactly generated.

A topological group without proper open subgroups trivially satisfies condition (ii) of Proposition 6] so from Corollary [1] we get

Corollary 13. A $\sigma$-compact metric group without proper open subgroups is compactly generated. 
Combining Corollaries 3 and 13 one gets:

Corollary 14. A $\sigma$-compact dense subgroup of a connected metric group is compactly generated.

It is easy to see that a topological group is dense in some connected group if and only if its completion with respect to the two-sided uniformity is connected.

It seems that even the following special case of Corollary 14 is new:

Corollary 15. A $\sigma$-compact connected metric group is compactly generated.

Recall that a topological group $G$ is totally bounded if $G$ is a subgroup of some compact group, or equivalently, if the completion of $G$ with respect to the (left, right or two-sided) group uniformity is compact [19]. An internal characterization of totally bounded groups says that a topological group $G$ is totally bounded iff for every open neighbourhood $V$ of the identity element of $G$ there exists a finite set $F \subseteq G$ with $G=F V$ [19]. It easily follows from this characterization that totally bounded groups satisfy condition (ii) of Proposition [6 and thus totally bounded groups are finitely generated modulo open sets. Therefore from Theorem 10 we obtain the following

Corollary 16. A $\sigma$-compact totally bounded metric group is compactly generated.

\section{SPECIFIC RESUlts FOR COUNTABle METRIC GROUPS}

Let $G$ be a topological group and $e$ its identity element. A sequence converging to $e$ is a map $s: \mathbf{N} \rightarrow G$ defined on the set $\mathbf{N}$ of natural numbers such that for every open set $U$ which contains $e$ there exists $n \in \mathbf{N}$ with $s(m) \in U$ for all $m \geq n$. In this section we take the liberty to identify a sequence $s: \mathbf{N} \rightarrow G$ with its image $S=\{s(n): n \in \mathbf{N}\}$. In particular, we will say that a topological group $G$ is algebraically generated by a sequence converging to its identity element if there exists a sequence $s: \mathbf{N} \rightarrow G$ converging to the identity element $e$ of $G$ such that its image $S=\{s(n): n \in \mathbf{N}\}$ algebraically generates $G$.

It is worth emphasizing that our definition does not exclude eventually constant sequences. In particular, a finitely generated topological group is algebraically generated by a (eventually constant) sequence converging to its identity element.

With this terminology in mind, our main result in this section is the following

Theorem 17. A countable metric group is compactly generated if and only if it is algebraically generated by a sequence converging to its identity element. (Eventually constant sequences are not excluded here.)

Proof. Since (the image of) a convergent sequence is compact, it suffices only to prove that a countable compactly generated metric group $G$ can be algebraically generated by a sequence converging to the identity element $e$ of $G$. Since $G$ is compactly generated, from Theorem 8 it follows that for every open subgroup $H$ of $G$ there exists a finite set $F$ such that $F \cup H$ algebraically generates $G$. Now all the assumptions of Theorem 9 are satisfied with $D=G$. Applying this theorem we can find a sequence $S$ converging to $e$ with $G=\langle S\rangle$.

Corollary 18. A countable metric group without proper open subgroups is generated by a (possibly eventually constant) sequence converging to its identity element.

Corollary 19. A countable dense subgroup of a connected metric group is generated by a (possibly eventually constant) sequence converging to its identity element. 
Corollary 20. A countable totally bounded metric group is generated by a (possibly eventually constant) sequence converging to its identity element.

It may be worth comparing the above three corollaries with the following result from [4, 2.2 and 2.3]: Every countable Hausdorff group $G$ has a closed (in $G$ ) discrete subset of $G \backslash\left\{1_{G}\right\}$ that algebraically generates $G$ (here $1_{G}$ is the identity element of $G$ ).

\section{What HAPPENS IN THE NONMETRIC CASE?}

In this section we will demonstrate that metrizability of the group is essential in most of our results.

We will use $A(X)$ to denote the free Abelian topological group of a Tychonoff space $X$ [12], [13] (see also [10, Ch. II, §8]), and $G(X)$ will be used to denote the subgroup of $A(X)$ consisting of all words $n_{1} x_{1}+\cdots+n_{k} x_{k}$ with $\sum_{i=1}^{k} n_{i}=0$. We first prove two general, albeit technical, facts about free Abelian topological groups.

Lemma 21. If a nonempty space $X$ is $\sigma$-compact, separable and connected, then so is $G(X)$.

Proof. 1 Let $\mathbf{Z}$ be the set of integers, and for each $k \in \mathbf{N}$ define

$$
S_{k}=\left\{\left(n_{1}, \ldots, n_{k}\right) \in \mathbf{Z}^{k}: \sum_{i=1}^{k} n_{i}=0\right\} .
$$

For $\left(n_{1}, \ldots, n_{k}\right) \in S_{k}$, define

$$
P\left(n_{1}, \ldots, n_{k}\right)=\left\{n_{1} x_{1}+\cdots+n_{k} x_{k}: x_{1} \in X, \ldots, x_{k} \in X\right\} \subseteq G(X) .
$$

Since $X$ is $\sigma$-compact, separable and connected, so are $X^{k}$ and $P\left(n_{1}, \ldots, n_{k}\right)$ because the latter space is a continuous image of $X^{k}$. Clearly,

$$
G(X)=\bigcup_{k \in \mathbf{N}} \bigcup_{\left(n_{1}, \ldots, n_{k}\right) \in S_{k}} P\left(n_{1}, \ldots, n_{k}\right) .
$$

Since the number of summands is at most countable, it follows that $G(X)$ is $\sigma$ compact and separable. To conclude that $G(X)$ is connected, it suffices to notice that $0 \in P\left(n_{1}, \ldots, n_{k}\right)$ for $k \in \mathbf{N}$ and $\left(n_{1}, \ldots, n_{k}\right) \in S_{k}$. Indeed, if $x \in X$ (such an $x$ exists since $X$ is nonempty), then

$$
0=0 x=\left(n_{1}+\cdots+n_{k}\right) x=n_{1} x+\cdots+n_{k} x \in P\left(n_{1}, \ldots, n_{k}\right) .
$$

Lemma 22. Assume that $X$ is a noncompact metric space and $Y$ is a dense subset of $X$. Let $H=G(X) \cap\langle Y\rangle$. Then no compactly generated subgroup of $H$ is dense in $H$.

Proof. Assume now that $H^{\prime}$ is a subgroup of $H$ algebraically generated by its compact subset $K^{\prime}$. Then there exists a compact subset $K$ of $X$ such that $K^{\prime} \subseteq$

\footnotetext{
${ }^{1}$ The proof of this lemma is included upon suggestion of the referee.
} 
$\langle K\rangle$ Thus $H^{\prime}=\left\langle K^{\prime}\right\rangle \subseteq\langle K\rangle$. Note that $\langle K\rangle$ is a closed subgroup of $A(X)$ [8], so the closure of $H^{\prime}$ in $H$ is included in $\langle K\rangle$. Using the assumption of our lemma we can pick two different points $x$ and $y$ from $Y \backslash\langle K\rangle=Y \backslash K$. Now observe that $x-y \in H \backslash\langle K\rangle$. Therefore, $H^{\prime}$ is not dense in $H$.

Our next example shows that "metric" cannot be omitted in Corollaries 14 and 15 Since connected groups do not have proper open subgroups (Corollary 3), Theorem 10 and Corollaries 11,12 and 13 become false if "metric" is omitted from their statements.

Example 23. There exists a separable, connected $\sigma$-compact Abelian group $G$ such that no compactly generated subgroup of $G$ is dense in $G$. In particular, $G$ itself is not compactly generated.

Proof. Let $\mathbf{R}$ be the space of real numbers with its usual metric topology. Take $G(\mathbf{R})$ as $G$.

From Lemma 21 it follows that $G$ is separable, $\sigma$-compact and connected. Taking $X=Y=\mathbf{R}$ in Lemma 22, we conclude that $G$ contains no dense compactly generated subgroup.

Our next example shows that "metric" is essential in Theorem 9 and Corollaries 18 and 19 .

Example 24. There exists a countable dense subgroup $H$ of a connected ( $\sigma$ compact Abelian) group such that no compactly generated subgroup of $H$ is dense in $H$. In particular, $H$ itself is not compactly generated.

Proof. Let $X=\mathbf{R}, Y=\mathbf{Q}$, where $\mathbf{R}$ is the space of real numbers with its usual metric topology and $\mathbf{Q}$ is its subspace consisting of rational numbers. Let $H$ be the group defined in Lemma 22. Obviously, $H$ is countable. Furthermore, $H$ is easily seen to be dense in $G(X)$. By Lemma 21$] G(X)$ is $\sigma$-compact and connected.

From Lemma 22 it follows that $H$ contains no dense compactly generated subgroups.

For an Abelian group $G$ (without any topology) let $G^{\#}$ denote the group $G$ equipped with the maximal totally bounded (Hausdorff) group topology (see [3] or [5] for the definition of this topology and the proof of its existence).

Lemma 25. For every Abelian group $G$, the group $G^{\#}$ is compactly generated if and only if $G$ is (algebraically) finitely generated.

Proof. We may assume, without loss of generality, that $G$ is infinite. All compact subsets of $G^{\#}$ are finite 3 So if $G^{\#}$ is compactly generated, it must be finitely generated 4

\footnotetext{
${ }^{2}$ This fact follows immediately from [2, Theorem 1.5 or Corollary 1.8]. The latter result is attributed by the authors of [2] to Arhangel'skii and Čoban, although Coban's proof contained a gap. It might be worth noting that Pestov [16, Proposition 6] points out that [2, Theorem 1.5] itself follows by applying Arhangel'skii's theorem [1 Proposition 2] to the spaces $X$ and $A(X)$, respectively, and to the correspondence $f \mapsto \bar{f}$, where $f: X \rightarrow \mathbf{R}$ and $\bar{f}$ is a homomorphism from $A(X)$ to $\mathbf{R}$, viewed as a linear map $C(X) \rightarrow C(A(X))$. The authors would like to thank Oleg Okunev and Vladimir Pestov for providing the information contained in this footnote.

${ }^{3}$ This was proven for the first time in [1] and then later reproven in [6, (1.3)]. The result also immediately follows from a much stronger fact that every infinite subset of $G^{\#}$ contains an infinite closed and relatively discrete subset [9].

${ }^{4}$ The referee has kindly suggested that the following comment should be added to our manuscript: "We used above the fact that $G^{\#}$ has only finite compact sets. This is a particular case
} 
Our next theorem demonstrates that "metric" cannot be omitted from Corollaries [16] and 20] Recall that a group $G$ is divisible if for every natural number $n$ and each element $g \in G$ there exists $h \in G$ so that $g=h^{n}$.

Theorem 26. Let $G$ be a countable Abelian divisible group which is not finitely generated (for example, the group of rational numbers $\mathbf{Q}$ will do). Then:

(i) the completion of $G^{\#}$ is compact and connected,

(ii) $G^{\#}$ is countable, thus trivially $\sigma$-compact, but

(iii) $G^{\#}$ is not compactly generated.

In particular, a countable (divisible) dense subgroup of a compact connected group need not be compactly generated.

Proof. Since $G^{\#}$ is totally bounded, the completion $H$ of $G^{\#}$ is a compact group. Since $G^{\#}$ is divisible, so is $H$ [20, Lemma 1]. Since divisible compact groups are connected [14, Corollary 2], it follows that $H$ is connected.

We do not know if "metric" is essential in Theorem 4 and Corollary 5

Question 27. Must a $\sigma$-compact group $G$ be compactly generated if one of the following conditions holds:

(i) $G$ has a dense compactly generated subgroup,

(ii) $G$ has a dense finitely generated subgroup,

(iii) $G$ is monothetic, i.e., contains a dense subgroup algebraically isomorphic to the group of integers $\mathbf{Z}$ ?

We also do not know if Theorem 17 holds in the nonmetric case:

Question 28. Can every countable, compactly generated group be algebraically generated by (possibly eventually constant) sequence converging to its identity element?

\section{ACKNOWLEDGEMENT}

The authors would like to thank the referee for her/his comments and suggestions.

\section{REFERENCES}

[1] A. V. Arhangel'skii, Linear homomorphisms of function spaces, Soviet Math. Dokl. 25 (1982), 852-855. MR 84f:54020

[2] A. Arhangel'skii, O. Okunev and V. Pestov, Free topological groups over metrizable spaces, Topology Appl. 33 (1989), no. 1, 63-76. MR 90h:22002

[3] W. W. Comfort and K. A. Ross, Topologies induced by groups of characters, Fund. Math. 55 (1964), 283-291. MR 30:183

[4] W. W. Comfort, S. A. Morris, D. Robbie, S. Svetlichny and M. Tkačenko, Suitable sets for topological groups, Topology Appl. 86 (1998), 25-46. MR 99g:54033

[5] W. W. Comfort and V. Saks, Countably compact groups and finest totally bounded topologies, Pacif. J. Math. 49 (1973), 33-44. MR 51:8321

of a more general phenomenon: Let $G$ be a locally compact Abelian group, and denote by $G^{+}$ the underlying group of $G$ equipped with the weakest topology that makes the (continuous in $G$ ) homomorphisms into the circle continuous. It follows that $G^{+}$is a totally bounded Hausdorff group such that $A \subseteq G$ is compact as a subspace of $G$ if and only if $A$ is compact as a subspace of $G^{+}$. This result was first obtained in [7, although the discrete version was obtained earlier in 11]. Regarding sequences, the reader must check [17]." 
[6] W. W. Comfort and F. Javier Trigos-Arrieta, Remarks on a theorem of Glicksberg, in: General Topology and Applications, Lecture Notes in Pure and Appl. Math., 134, New York (1991), 25-33. MR 92k:54042

[7] I. Glicksberg, Uniform boundedness for groups, Canadian J. Math. 14 (1962), 269-276. MR 27:5856

[8] M. I. Graev, Free topological groups, Izvestiya Akad. Nauk SSSR, Ser. Mat. 12 (1948), 279324 (in Russian); English transl. in: Topology and Topological Algebra, Amer. Math. Soc. Translation Ser. 1, vol. 8 (1962), 305-364. MR 10:11d

[9] K. P. Hart and J. van Mill, Discrete sets and the maximal totally bounded group topology, J. Pure Appl. Algebra 70 (1991), 73-80. MR 92c:20101

[10] E. Hewitt and K. Ross, Abstract harmonic analysis, vol. I, Springer-Verlag (1963), Berlin. MR 28:158

[11] Horst Leptin, Abelsche Gruppen mit kompakten Charaktergruppen und Dualitätstheorie gewisser linear topologischer abelscher Gruppen, Abh. Math. Sem. Univ. Hamburg 19 (1955), 244-263. MR 16:899f

[12] A. A. Markov, On free topological groups, Doklady Akad. Nauk SSSR 31 (1941), 299-301 (in Russian)

[13] A. A. Markov, Three papers on topological groups, Amer. Math. Soc. Transl. 30 (1950), 120 pp. MR 12:318b

[14] J. Mycielski, Some properties of connected compact groups, Colloq. Math. 5 (1958), 162-166. MR 20:6479

[15] V. G. Pestov, On compactly generated topological groups, Mat. Zametki 40 (1986), 671-676 (in Russian); English transl. in: Math. Notes 40 (1986), 880-882. MR 88j:22002

[16] V. Pestov, Free abelian topological groups and the Pontryagin-van Kampen duality, Bull. Austral. Math. Soc., 52, no. 2 (1995), 297-311. MR 96k:22002

[17] G. A. Reid, On sequential convergence in groups, Math. Zeitschrift 102 (1967), 227-235. MR 36:3069

[18] T. C. Stevens, Connectedness of complete metric groups, Colloq. Math. 50 (1986), 233-240. MR 88h:54056

[19] A. Weil, Sur les espaces à structure uniforme et sur la Topologie Générale, Hermann, Paris (1937).

[20] H. Wilcox, Dense subgroups of compact groups, Proc. Amer. Math. Soc. 28 (1971), 578-580. MR 43:6359

Department of Mathematical Sciences, Faculty of Science, Ehime University, MatSUYAMA 790-8577, JAPAN

E-mail address: fujita@math.sci.ehime-u.ac.jp

Department of Mathematical Sciences, Faculty of Science, Ehime University, MatSUYAMA 790-8577, JAPAN

E-mail address: dmitri@dpc.ehime-u.ac.jp 